\title{
Joint Closed-Loop Power Control and Adaptive Beamforming for Wireless Networks with Antenna Arrays in a 2D Urban Environment
}

\author{
Mohamad Dosaranian Moghadam ${ }^{1}$, Hamidreza Bakhshi², Gholamreza Dadashzadeh² \\ ${ }^{1}$ Department of Electrical Engineering, Islamic Azad University, Qazvin Branch, Qazvin, Iran \\ ${ }^{2}$ Department of Electrical Engineering, Shahed University, Tehran, Iran \\ E-mail:m_dmoghadam@qiau.ac.ir,bakhshi@shahed.ac.ir,gdadashzadeh@shahed.ac.ir \\ Received July 25, 2010; revised August 31, 2010; accepted October 2, 2010
}

\begin{abstract}
The interference reduction capability of antenna arrays and the power control algorithms have been considered separately as means to decrease the interference in wireless communication networks. In this paper, we propose smart step closed-loop power control (SSPC) algorithm in wireless networks in a 2D urban environment with constrained least mean squared (CLMS) algorithm. This algorithm is capable of efficiently adapting according to the environment and able to permanently maintain the chosen frequency response in the look direction while minimizing the output power of the array. Also, we present switched-beam (SB) technique for enhancing signal to interference plus noise ratio (SINR) in wireless networks. Also, we study an analytical approach for the evaluation of the impact of power control error (PCE) on wireless networks in a 2D urban environment. The simulation results indicate that the convergence speed of the SSPC algorithm is faster than other algorithms. Also, we observe that significant saving in total transmit power (TTP) are possible with our proposed algorithm. Finally, we discuss three parameters of the PCE, number of antenna elements, and path-loss exponent and their effects on capacity of the system via some computer simulations.
\end{abstract}

Keywords: Adaptive Beamforming, Closed-Loop Power Control, Matched Filter, Urban Signal Propagation Simulator, Power Control Error

\section{Introduction}

Diversity and power control are two effective techniques for enhancing the signal to interference plus noise ratio (SINR) for wireless networks. Diversity exploits the random nature of radio propagation by finding independent (or, at least, highly uncorrelated) signal paths for communication. If one radio path undergoes a deep fade, another independent path may have a strong signal. By having more than one path to select from, the SINR at the receiver can be improved. The diversity scheme can be divided into three methods: 1 ) the space diversity; 2) the time diversity; 3 ) the frequency diversity. In these schemes, the same information is first received (or transmitted) at different locations (or time slots/frequency bands). After that, these signals are combined to increase the received SINR. The antenna array is an example of the space diversity, which uses a beamformer to increase the SINR for a particular direction [1]. Accordingly, the use of smart antennas is expected to have a significant impact on future wireless communications to meet the projected perspective of future communication networks. A major reason to use smart antenna in wireless communication is its capability to intelligently respond to the unknown interference environment in real time. The process of formation of nulls in the direction of interference and strong beams in the direction of desired user is called adaptive array processing. These systems are called adaptive beamforming system and consist of spatially disposed sensor elements connected to a single channel or to a multi channel adaptive processor. The term adaptive beamforming is also referred as smart antennas. Adaptive antenna array can be used to eliminate the directional interference by adaptive canceling and therefore to improve the SINR. Steering capability of adaptive array depends on processing algorithms for null steering. Such algorithms are called adaptive algorithm. In wireless communications, smart antennas are used due to 
their ability to separate the desired signal from interfering signals. By knowing the direction of the desired signals, they are able to adjust the antenna pattern intelligently by adjusting the weights of the adaptive algorithm [2-4].

On the other hand, power control can be realized by allocating different power levels to the links with different link gains. For example, links with smaller links gains are supposed to have larger power levels. This larger power level may cause more interference to other users. Therefore, adequate power control is needed to meet the SINR requirement while minimizing the power consumption and increasing the capacity in all network. In general, two types of power control are often considered. 1) Closed-loop power control 2) Open-loop power control [2,3]. In a closed-loop power control, according to the received signal power at a base station (BS), the base station sends a command to a mobile set to adjust the transmit power of the mobile. Also, closed-loop power control is employed to combat fast channel fluctuations due to fading. Closed-loop algorithms can effectively compensate fading variations when the power control updating time is smaller than the correlation time of the channel. However, in an open-loop power control, a mobile user adjusts its transmit power according to its received power in downlink [1-6].

The first goal of this paper is to extend our work in [7,8] by considering closed-loop power control and power control error (PCE). In [7], a constrained least mean squared (CLMS) algorithm was used for tracking mobile user in a 2D urban environment without power control algorithm. Also in [8], we proposed the SSPC algorithm in wireless network by the CLMS algorithm without considering the effect of PCE in 2D urban environment. Accordingly, in this paper we present smart step closed-loop power control (SSPC) algorithm for minimizing the total transmit power (TTP) in wireless networks [9]. On the other hand, the issue of the effect of power control errors on wireless networks has received a great deal of attention over the last few years [2,3]. Finally, we consider the effect of PCE on wireless networks in a 2D urban environment [10].

The organization of the remainder of this paper is as follows. In Section 2, propagation model in a 2D urban environment and also functional state of urban signal propagation simulator (USPS) are described. Section 3 considers the adaptive antenna array. The CLMS algorithm is summarized in Section 4. In Section 5, we present the SSPC algorithm. Then in Section 6, we extend the analysis to the case of PCE on wireless networks. Section 7 presents switched-beam (SB) technique and equal sectoring (ES) method. Finally, simulation results and conclusions are given in Sections 8 and 9.

\section{Propagation Model}

Because of using 2D urban structure in reverse link (uplink) in this work, for computing yield for path between a user and BS, propagation model in urban environments are dramatized. In propagation model in urban environments and in reverse link, user antenna is radiating beams which are diffusing in all directions and parts of beams reach to $\mathrm{BS}$.

In urban environment, delivered beam from user by the time of collision to an obstacle like a wall surface or a building, reflects to a new angle and continues its path, this is called reflection phenomena. In condition when radiated beam is conflicted to a obstacle edge then diffraction phenomena is happened and diffracting point is diffusing new beams to all directions like a transmitter. All reflected beams, will stay in the environment till the time their power are not reduced to a threshold limit. Figure 1 shows both phenomena in reverse link. In this work, USPS dramatizing is used for implementing a 2D urban environment $[7,11]$.

According to above dramatization, we could see because of line of sight (LoS) in un-urban environment, only one signal is delivered from each user to receiver, while in function and because of elimination phenomena in an urban environment, beside to signals which are delivered to line sight, signals which have difference in phase or domain with this signal are also received by receiver.

\section{Adaptive Antenna Array}

An adaptive antenna array consists of a set of antennas, designed to receive signals radiating from some specific directions and attenuate signals radiating from other directions of no interest. The outputs of array elements are weighted and added by a beamformer, as shown in Figure 2 , to produce a directed main beam and adjustable

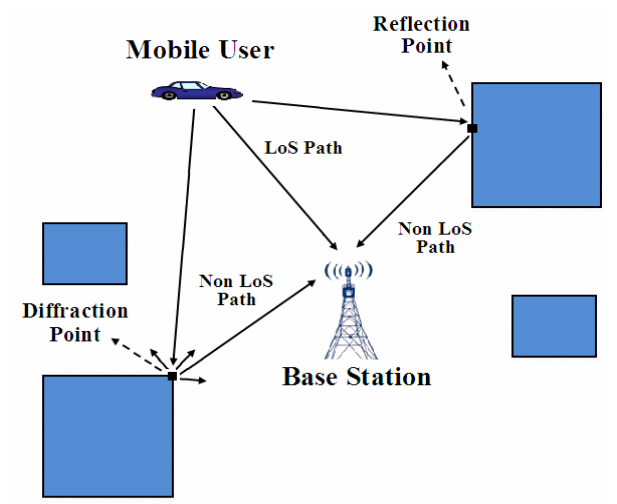

Figure 1. Diffraction phenomena and reflection phenomena (LoS and Non-LoS paths) for a 2D urban environment in reverse link. 


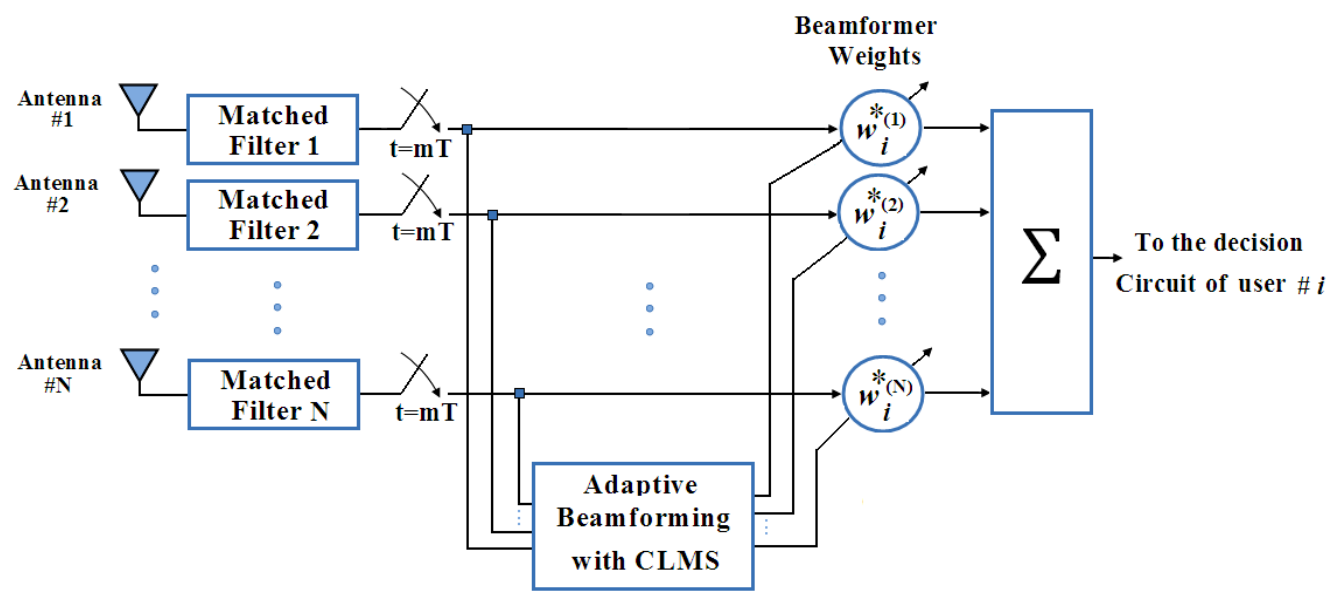

Figure 2. Antenna array and beamformer [13].

nulls. In order to reject the interference, the beamformer has to place its nulls in the directions of sources of interference, and steer to the direction of the target signal by maintaining constant gain at this direction [12]. In this paper, we focus on the uplink communication path in a single-cell wireless system with $M$ users and only one BS. Also we use a uniform linear array (ULA) of $N$ antenna elements.

Accordingly, the received signal vector in BS can be written as [12]

$$
\mathbf{x}(t)=\sum_{i=1}^{M} \sqrt{p_{i}^{\prime}} s_{i}\left(t-\tau_{i}\right) \mathbf{v}_{i}(\theta)+\mathbf{n}(t)
$$

where $s_{i}(t)$ is the message signal transmitted from the $i$ th user; $p_{i}^{\prime}$ is the received power of the $i$ th user in BS which is equal to $p_{i} G_{i}$ where $p_{i}$ and $G_{i}$ denote the transmitted power of the $i$ th user, and the link gain between user $i$ and BS in LoS path (or the best non-LoS path if LoS path is not exist), as shown in Figure 1, respectively. Also $\mathbf{n}(t)$ is the thermal noise vector at the input of antenna array at BS and $\tau_{i}$ is the corresponding time delay. For simplicity, we further assume that the time delays for all uses are equal to 0 . Also the $N \times 1$ vector $\mathbf{v}_{i}(\theta)$ is the array response for user $i$ and can be written as follows [12]:

$$
\mathbf{v}_{i}(\theta)=\sum_{l=1}^{L_{i}} \sqrt{\alpha_{i, l}} \mathbf{a}_{i}\left(\theta_{i, l}\right)
$$

where $L_{i}$ is the number of paths between user $i$ and BS in USPS; $\theta_{i, l}$ is the direction of arrival (DoA) in the Ith path for user $i ; \alpha_{i, l}$ is the normalized attenuation by the best link gain $\left(G_{i}\right)$ in USPS between user $i$ and BS in the lth path, therefore $0<\alpha_{i, l} \leq 1$. Also, response of the base station array to the direction $\theta_{i, l}$ is given by

$$
\mathbf{a}_{i}\left(\theta_{i, l}\right)=\left[1 \exp \left(-j \tau_{\theta_{i, l}}\right) \ldots \exp \left(-j(N-1) \tau_{\theta_{i, l}}\right)\right]^{T}
$$

where $j=\sqrt{-1}$ and $\tau_{\theta_{i, l}}=(2 \pi d / \lambda) \sin \theta_{i, l}$, in which $\lambda$ is signal wavelength and $d$ is the distance between the antenna elements that for avoid the spatial aliasing should be defined as $d=0.5 \lambda$.

In non-spread spectrum systems, the transmitted signal is given by [12]

$$
s_{i}(t)=\sum_{m} b_{i}(m) g(t-m T)
$$

where $b_{i}(m)$ is the ith user information bit stream with a bit period of $T$ and $g(t)$ is the pulse-shaping filter impulse response. It has been shown that the output of a matched filter sampled at the symbol intervals is a sufficient statistic for the estimation of the transmitted signal. The matched filter is sampled at $t=m T$

$$
\mathbf{x}(m)=\left.\mathbf{x}(t) * g^{*}(-t)\right|_{t=m T}
$$

Hence, the received signal at the output of the matched filter is given by

$$
\begin{aligned}
& \mathbf{x}(m)=\sqrt{p_{i} G_{i}} b_{i}(m) \mathbf{v}_{i}(\theta)+\sum_{\substack{k=1 \\
k \neq i}}^{M} \sqrt{p_{k} G_{k}} b_{k}(m) \mathbf{v}_{k}(\theta)+ \\
& \mathbf{n}(m)=\sqrt{p_{i} G_{i}} b_{i}(m) \mathbf{v}_{i}(\theta)+\mathbf{I}_{i}(m)+\mathbf{n}(m)
\end{aligned}
$$

where $\mathbf{I}_{i}(m)$ is the interference for user $i$ in BS and $\mathbf{n}(m)=\left.\mathbf{n}(t) * g^{*}(-t)\right|_{t=m T}$.

\section{Constrained LMS Algorithm}

Constrained LMS algorithm is a gradient based algorithm to minimize the total processor output power, based on the look direction constraint. The adaptive algorithm is designed to adapt efficiently in agreement with the environment and able to permanently preserve the desired frequency response in the look direction while minimizing the output power of the array. The 
combined form of the constraint is called constraint for narrowband beamforming [12-14].

This form consider a narrowband beamformer where the narrowband signal from each element of smart antenna are multiplied by the complex weight calculated by using narrowband adaptive beamforming algorithm, and then summed to produce the output of the array. The definition of the complex weights of this beamformer in the $m$ th iteration for the ith user is as follows [13-15]:

$$
\mathbf{w}_{i}(m)=\left[w_{i}^{(1)}(m) w_{i}^{(2)}(m) \ldots w_{i}^{(N)}(m)\right]^{T}
$$

Accordingly, the output of the array in the $m$ th iteration for user $i$ is given by

$$
y_{i}(m)=\mathbf{w}_{i}^{H}(m) \mathbf{x}(m)
$$

The expected output power of the array in the $m$ th iteration is given by

$$
\begin{aligned}
& \mathrm{E}\left(\left|y_{i}(m)\right|^{2}\right)=\mathrm{E}\left(y_{i}(m) y_{i}^{*}(m)\right) \\
& \quad=\mathrm{E}\left(\mathbf{w}_{i}^{H}(m) \mathbf{x}(m) \mathbf{x}^{H}(m) \mathbf{w}_{i}(m)\right)=\mathbf{w}_{i}^{H}(m) \mathbf{R}_{x x} \mathbf{w}_{i}(m)
\end{aligned}
$$

where $\mathrm{E}($.$) is denoted the expectation and \mathbf{R}_{x x}$ is the correlation matrix of the received signal vector $\mathbf{x}(m)$.

A real-time CLMS algorithm for determining the optimal weight vector for user $i$ is $[13,14]$ :

$$
\left\{\begin{array}{l}
\mathbf{w}_{i}(m+1)=\mathbf{w}_{i}(m)+\mu g\left(\mathbf{w}_{i}(m)\right) \\
\mathbf{w}_{i}^{H} \mathbf{a}_{i}\left(\theta_{i, g_{\max }}\right)=1
\end{array}\right.
$$

where $\mathbf{a}_{i}\left(\theta_{i, \mathrm{~g}_{\max }}\right)$ denotes spatial response of the array for user $i$ in the best path gain, i.e., $\alpha_{i, l}=1$, and $\mathbf{w}_{i}(m+1)$ is the new weight computed at the $(m+1)$ th iteration for user $i$. Also, the variable scalar $\mu$ denotes a positive scalar (gradient step size) that controls the convergence characteristic of the algorithm, that is, how fast and how close the estimated weights approach the optimal weights, and $g\left(\mathbf{w}_{i}(m)\right)$ denotes an unbiased estimate of the gradient of the power surface $\left(\mathbf{w}_{i}(m)^{H} \mathbf{R}_{x x} \mathbf{w}_{i}(m)\right.$ which is the expected output power of the array) with respect to $\mathbf{w}_{i}(m)$ after the mth iteration. The algorithm is "constrained" because the weight vector satisfies the constraint at each iteration, that is $\mathbf{w}_{i}^{H} \mathbf{a}_{i}\left(\theta_{i, g_{\max }}\right)=1$. Rewrite the CLMS algorithm as follows [14].

$$
\mathbf{w}_{i}(m+1)=\boldsymbol{\beta}_{i}\left(\mathbf{w}_{i}(m)+\mu g\left(\mathbf{w}_{i}(m)\right)\right)+\frac{\mathbf{a}_{i}\left(\theta_{i, g_{\max }}\right)}{N}
$$

where

$$
\boldsymbol{\beta}_{i}=\mathbf{I}-\frac{\mathbf{a}_{i}\left(\theta_{i, g_{\max }}\right) \mathbf{a}_{i}\left(\theta_{i, g_{\max }}\right)^{H}}{N}
$$

The gradient of $\mathbf{w}_{i}^{H}(m) \mathbf{R}_{x x} \mathbf{w}_{i}(m)$ with respect to $\mathbf{w}_{i}(m)$ is given by

$$
g\left(\mathbf{w}_{i}(m)\right) \stackrel{\Delta}{=}-\frac{\partial}{\partial \mathbf{w}_{i}^{*}}\left(\mathbf{w}_{i}^{H}(m) \mathbf{R}_{x x} \mathbf{w}_{i}(m)\right)=-2 \mathbf{R}_{x x} \mathbf{w}_{i}(m)
$$

and its computation using this expression requires knowledge of $\mathbf{R}_{x x}$, which normally is not available in practice. For a standard LMS algorithm, an estimate of the gradient at each iteration is made by replacing $\mathbf{R}_{x x}$ by its noise sample $\mathbf{x}_{i}(m+1) \mathbf{x}_{i}(m+1)^{H}$ available at time instant $(m+1)$, leading to

$$
g\left(\mathbf{w}_{i}(m)\right)=-2 \mathbf{x}_{i}(m+1) y_{i}^{*}(m)
$$

The CLMS is a fast convergence algorithm. However, it is drastically sensitive to the mismatch in the direction of arrival. Meanwhile, the weights estimated by the standard algorithm are sensitive to the signal power, requiring a lower step size in the presence of a strong signal for the algorithm to converge, which in turn regarding the decrease of mis-adjustment error, the convergence time is increased [14-16].

Consider the problem of beamforming as to maximize the SINR for a specific link, which is equivalent to minimizing the interference at the receiver of that link. In order to minimize the variance or average power at the output of the beamformer subject to maintaining unity gain at the direction of the mobile user signal. Accordingly, we can rewrite the average output power for user $i$ as [12]

$$
\zeta_{i}=\hat{\mathbf{w}}_{i}^{H} \mathbf{R}_{x x} \hat{\mathbf{w}}_{i}
$$

where $\hat{\mathbf{w}}_{i}$ is the optimum weight vector for user $i$ with CLMS algorithm. If the message signals $s_{i}(t)$ are uncorrelated and zero mean, the correlation matrix $\mathbf{R}_{x x}$ is given by

$$
\begin{aligned}
\mathbf{R}_{x x}= & p_{i} G_{i} \mathbf{v}_{i}(\theta) \mathbf{v}_{i}^{H}(\theta)+\sum_{\substack{k=1 \\
k \neq i}}^{M} p_{k} G_{k} \mathbf{v}_{k}(\theta) \mathbf{v}_{k}^{H}(\theta)+\sigma_{n}^{2} \mathbf{I} \\
& =p_{i} G_{i} \mathbf{v}_{i}(\theta) \mathbf{v}_{i}^{H}(\theta)+\boldsymbol{\Phi}_{i}
\end{aligned}
$$

where

$$
\boldsymbol{\Phi}_{i}=\sum_{\substack{k=1 \\ k \neq i}}^{M} p_{k} G_{k} \mathbf{v}_{k}(\theta) \mathbf{v}_{k}^{H}(\theta)+\sigma_{n}^{2} \mathbf{I}
$$

is the correlation matrix of unwanted signals for user $i$ in BS, and $\sigma_{n}^{2}$ is the noise power at the input of each array element.

Combining (15)-(17), we obtain the received signal plus interference power as a function of weight vector

$$
\begin{gathered}
\zeta_{i}=p_{i} G_{i}\left|\hat{\mathbf{w}}_{i}^{H} \mathbf{v}_{i}(\theta)\right|^{2}+\sum_{\substack{k=1 \\
k \neq i}}^{M} p_{k} G_{k} \hat{\mathbf{w}}_{i}^{H} \mathbf{v}_{k}(\theta) \mathbf{v}_{k}^{H}(\theta) \hat{\mathbf{w}}_{i} \\
+\sigma_{n}^{2} \hat{\mathbf{w}}_{i}^{H} \hat{\mathbf{w}}_{i}
\end{gathered}
$$


Accordingly, the SINR at BS for user $i$ can be written as follows [12].

$$
\operatorname{SINR}_{i}(\hat{\mathbf{w}}, \mathbf{v}(\theta))=\frac{p_{i} G_{i}\left|\hat{\mathbf{w}}_{i}^{H} \mathbf{v}_{i}(\theta)\right|^{2}}{\sum_{\substack{k=1 \\ k \neq i}}^{M} p_{k} G_{k}\left|\hat{\mathbf{w}}_{i}^{H} \mathbf{v}_{k}(\theta)\right|^{2}+\sigma_{n}^{2} \hat{\mathbf{w}}_{i}^{H} \hat{\mathbf{w}}_{i}}
$$

In order to perform the bit error rate (BER), we assume Gaussian approximation for the probability density function of interference plus noise. The conditional BER for a BPSK modulation is [17]:

$$
\operatorname{BER}_{i}(\hat{\mathbf{w}}, \mathbf{v}(\theta))=Q\left(\sqrt{2 \times \operatorname{SINR}_{i}(\hat{\mathbf{w}}, \mathbf{v}(\theta))}\right)
$$

where

$$
Q(x)=\frac{1}{\sqrt{2 \pi}} \int_{x}^{\infty} \exp \left(-u^{2} / 2\right) d u
$$

It should be mentioned that for the array antenna weight vector elements in the CLMS algorithm and for big $\mu$, will converge after a few iteration (is approximately equal to the number of beamformer weights, i.e., $m=N$ ) $[7,16]$. Moreover it is obvious that, without power control algorithm, the calculated optimal weight vector does not guarantee desirable SINR for the mobile user. Thus, we introduce our proposed algorithm in next section.

\section{Smart Step Closed-Loop Power Control Algorithm}

A major limiting factor for the satisfactory performance of cellular systems is the near-far effect. Power control is an intelligent way of adjusting the transmitted powers in cellular systems so that the TTP is minimized, but at the same time, the user SINRs satisfies the system quality of service (QoS) requirements, [18,19].

Depending on the location where the decision on how to adjust the transmitted powers is made, the power control algorithms can be divided into two groups: centralized and distributed techniques [1-6,12]. In centralized power control, a network center can simultaneously compute the optimal power levels for all users. However, it requires measurement of all the link gains and the communication overhead between a network center and base stations. Thus, it is difficult to realize in a large system [20]. Distributed power control, on the other hand, uses only local information to determine transmitter power levels. It is much more scalable than centralized power control. However, transmitter power levels may not be optimal, resulting in performance degradation [21,22].

The distributed closed-loop power control problem has been investigated by many researchers from many perspectives during recent years [5,18,21-23]. For instance, distributed conventional closed-loop power control strategy used in practice in wireless cellular systems as code division multiple access (CDMA) is a fixed-step controller based on SINR measurements. The fixed-step power control (FSPC) algorithm is defined by [5]

$$
p_{i}^{n+1}=p_{i}^{n}+\delta \operatorname{sign}\left(\gamma_{i}^{*}-\gamma_{i}^{n}\right)
$$

Where $p_{i}^{n}, \gamma_{i}^{*}$ and $\gamma_{i}^{n}$ are the transmitter power, SINR target and measured SINR of user $i$ at time $n$, respectively, and $\delta$ is the fixed step size. Also $p_{i}^{n+1}$ is transmitter power control (TPC) command in the feedback link of BS to user $i$ at time $n+1$ (all signals are in decibels).

Also, the distributed traditional closed-loop power control (DTPC) is defined by [18]

$$
p_{i}^{n+1}=\frac{\gamma_{i}^{*}}{\gamma_{i}^{n}} p_{i}^{n}
$$

In both algorithms, the simple intuition behind this iteration is that if the current SINR $\gamma_{i}^{n}$ of user $i$ is less than the target SINR $\gamma_{i}^{*}$, then the power of that user is increased; otherwise, it is decreased.

It should be mentioned that convergence speed of DTPC algorithm is higher than FSPC algorithm. Also, the variance of the SINR mis-adjustment in FSPC algorithm is higher than DTPC algorithm. But, it has been shown that the FSPC algorithm converges to $\left|\gamma_{i}^{*}-\gamma_{i}^{n}\right| \leq 2 \delta k_{d}$, where $k_{d}$ is the loop delay [4].

Also in [23], variable step closed-loop power control (VSPC) algorithm has been proposed. In this algorithm, variable step size is discrete with mode $q$. It is shown that the performance of VSPC algorithm with mode $q=$ 4 is found to be worse than that of a fixed step algorithm $(q=1)$ under practical situations with loop delay of two power control intervals, but the convergence speed of VSPC algorithm is higher than FSPC algorithm. Also in this algorithm, the variance of the SINR mis-adjustment is reduced in compared to FSPC algorithm.

Practical implementations of power control in the CDMA cellular systems utilize closed-loop control, where the transmitter adjusts its power based on commands received from the receiver in a feedback channel. To minimize signaling overhead, typically one bit is used for the power control command. In practice, the command must be derived based on measurements made at the receiver, transmitted over the feedback channel to the transmitter, and finally processed and applied at the transmitter. All these operations constitute a loop delay, which can cause problems if it is not properly taken care of in the design of the power control algorithm. In many cases the loop delay is known due to a specific frame structure inherent in the system. A typical loop delay situation encountered in WCDMA systems is shown in 
Figure 3. The slot at time $n t$ is transmitted using power $p^{n}$. The receiver measures SINR $\gamma^{n}$ over a number of pilots and/or symbols and derives a TPC command. The command is transmitted to the transmitter in the feedback link and the transmitter adjusts its power at time $(n+1) t$ according to the command. It should be mentioned that since the power control signaling is standardized, the loop delays are known exactly [5].

In this paper, we propose smart step closed-loop power control algorithm for wireless networks. We express the SSPC algorithm as follows $[8,9]$.

$$
p_{i}^{n+1}=p_{i}^{n}+\delta\left|\gamma_{i}^{*}-\gamma_{i}^{n}\right| \operatorname{sign}\left(\gamma_{i}^{*}-\gamma_{i}^{n}\right)
$$

The algorithm is implemented as follows.

1) Select the initial transmitted power vector $(n=0)$ for all users as $\mathbf{p}^{0}=\left[\begin{array}{lll}p_{1}^{0} & p_{2}^{0} \ldots p_{M}^{0}\end{array}\right]$.

2) Estimate the weight vector for all users ( $\hat{\mathbf{w}}_{i}, 1 \leq i \leq M$ ) with the CLMS algorithm using (11).

3) Calculate the SINR for all users $(1 \leq i \leq M)$ at time $n$ and after the time $m=N$, according to the following equation:

$$
\gamma_{i}^{n}=\frac{p_{i}^{n} G_{i}\left|\hat{\mathbf{w}}_{i}^{H} \mathbf{v}_{i}(\theta)\right|^{2}}{\sum_{\substack{k=1 \\ k \neq i}}^{M} p_{k}^{n} G_{k}\left|\hat{\mathbf{w}}_{i}^{H} \mathbf{v}_{k}(\theta)\right|^{2}+\sigma_{n}^{2} \hat{\mathbf{w}}_{i}^{H} \hat{\mathbf{w}}_{i}}
$$

4) If $\left|\gamma_{i}^{*}-\gamma_{i}^{n}\right|>\varepsilon_{0}$ for each user then set $n=n+1$ and calculate the TPC for all users at time $n+1$ using (24) and go back to 2), where $\varepsilon_{0}$ is threshold value.

5) Finally, if $\left|\gamma_{i}^{*}-\gamma_{i}^{n}\right|<\varepsilon_{0}$ for all users then algorithm ends.

As will be seen from simulation results, because of variable coefficient in the sign function, the convergence speed of our algorithm is higher than FSPC and VSPC algorithms.

\section{Power Control Error}

When imperfections in power control are considered, multipath fading is not perfectly compensated. As a result, the power received from a mobile will not be constant at the base station to which the mobile is connected. Accordingly, we can be rewritten (1) as follows.

$$
\mathbf{x}(t)=\sum_{i=1}^{M} \sqrt{P \lambda_{i}} s_{i}\left(t-\tau_{i}\right) \mathbf{v}_{i}(\theta)+\mathbf{n}(t)
$$

where $P=E_{b} / T_{b}$ represents the received signal power of all users in the presence of PCE and $E_{b}$ is the energy per bit for all users. The variable $\lambda_{i}$ is PCE for user $i$, which is assumed to follow a log-normal distribution and thus it can be written as $\lambda_{i}=10^{v_{i} / 10}$, where $v_{i}$ is a Gaussian random variable with mean 0 and variance $\sigma_{v}^{2}$ for all users [3]. On the other hand, $E\left[\lambda_{i}\right]$ for all users can be written as follows [24].

$$
\mathrm{E}\left[\lambda_{i}\right]=e^{\beta^{2} \sigma_{v}^{2} / 2}
$$

where $\beta=\ln (10) / 10$. Accordingly, we can be rewritten the SINR in (19) as follows [10].

$$
\begin{aligned}
\operatorname{SINR}_{i}\left(\hat{\mathbf{w}}, \mathbf{v}(\theta), \sigma_{v}^{2}\right) & \frac{P e^{\beta v_{i}}\left|\hat{\mathbf{w}}_{i}^{H} \mathbf{v}_{i}(\theta)\right|^{2}}{P e^{\beta^{2} \sigma_{v}^{2} / 2} \sum_{\substack{k=1 \\
k \neq i}}^{M}\left|\hat{\mathbf{w}}_{i}^{H} \mathbf{v}_{k}(\theta)\right|^{2}+\sigma_{n}^{2} \hat{\mathbf{w}}_{i}^{H} \hat{\mathbf{w}}_{i}}
\end{aligned}
$$

\section{Switched-Beam Technique and Equal Sectoring Method}

One simple alternative to the fully adaptive antenna is the switched-beam architecture in which the best beam is chosen from a number of fixed steered beams. Switched-beam systems are technologically the simplest

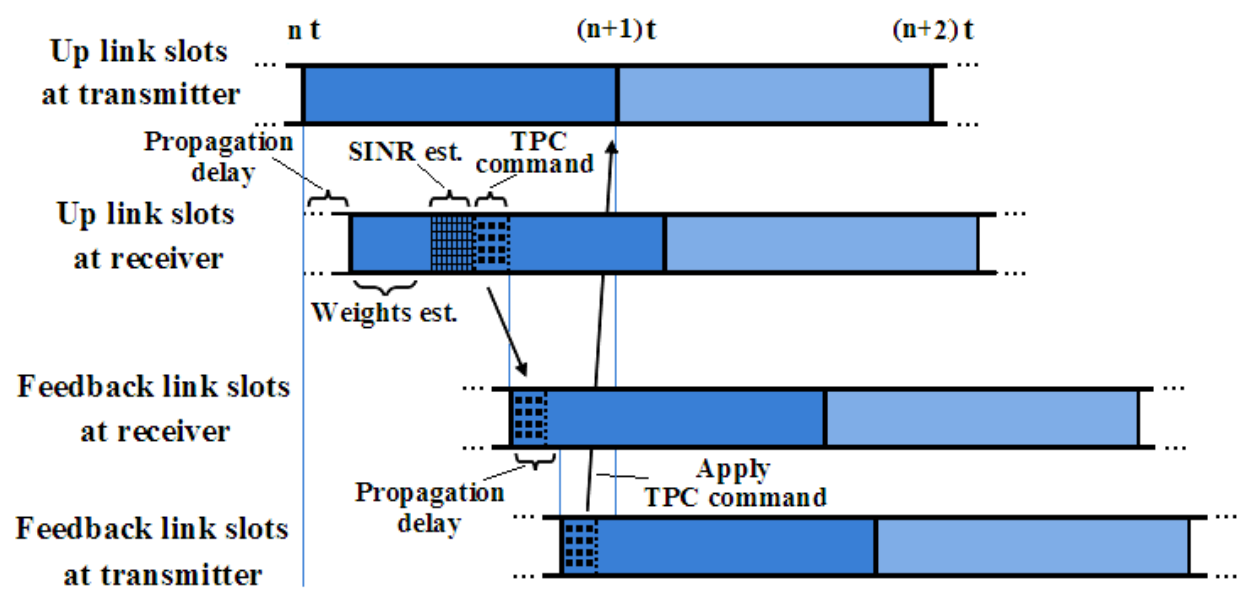

Figure 3. Example of power control timing in WCDMA systems [4]. 
and can be implemented by using a number of fixed, independent, or directional antennas [25]. We list the conditions of the SB technique for this paper as follows [11].

1) According to Figure 4, beams coverage angle is $30^{\circ}$ and overlap between consecutive beams is $20^{\circ}$. Thus each base station has 36 beams.

2) According to Figure 5, each user can be use one beam for its each path to communicate with a base station at any time.

One simple method to sectorize a cell is equal sectoring; in which all sectors have the same coverage angle. In this paper, we assume three sectors for each base station with sector angle $120^{\circ}$ for the ES method [26].

\section{Simulation Results}

In this section, we evaluate the performance of our algorithm by simulating the same system as in [7] with CLMS algorithm, SB technique, and ES method. For this purpose, we use part of two-dimensional map of the University of Toronto campus area in Canada as shown in Figure 6 [7]. According to this figure, we observe beside desired user, $M-1$ interference users in a stable positioning situation with uniform distribution are also

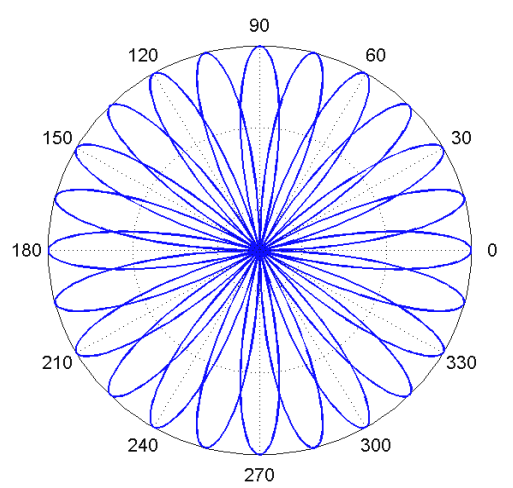

Figure 4. 36 beams in BS with the SB technique.



Figure 5. Select of beam for two users in two different paths with the SB technique. sending information to BS equipped to an array antenna in reverse link. According to the description in Section 2, sending information by all users to BS is performed by dramatizer USPS. Also note that the variance of the noise $\left(\sigma_{n}^{2}\right)$ for every element is assumed to be equal to 0.01 ; resolution and path loss in USPS $R=1$ and $L=0.05 \mathrm{~dB} / \mathrm{m}$, respectively; gradient step size in the CLMS algorithm $\mu=0.005$; fixed step size for SSPC, FSPC, and VSPC algorithms $\delta=0.05$; mode $q=4$ for VSPC algorithm [23]; threshold value in SSPC algorithm $\varepsilon_{0}=0.1 \mathrm{~dB}$; number of elements antenna array $N=10$; initial value for transmitted power vector for all users $\mathbf{p}^{0}=0$; and the SINR target value is the same for all users and is set to $\gamma^{*}=8(9 \mathrm{~dB})$. It also is assumed that the distribution of users is uniform.

Figure 7 shows the comparison of the average SINR achieved over $M=15$ users versus the power control iteration index $(n)$ for SSPC, VSPC ( $q=4)$, and FSPC algorithms and CLMS, SB, and ES techniques. Also in this simulation, we assume that each user to have a maximum power constraint of 1watt. It should be mentioned that in case of without power control (PC), the transmitted power for all users is 1 watt [7]. Accordingly, we observe that the convergence speed of the SSPC al-



Figure 6. Two-dimensional map of the University of Toronto campus area and placing users and base station contain array antenna in map center [7].

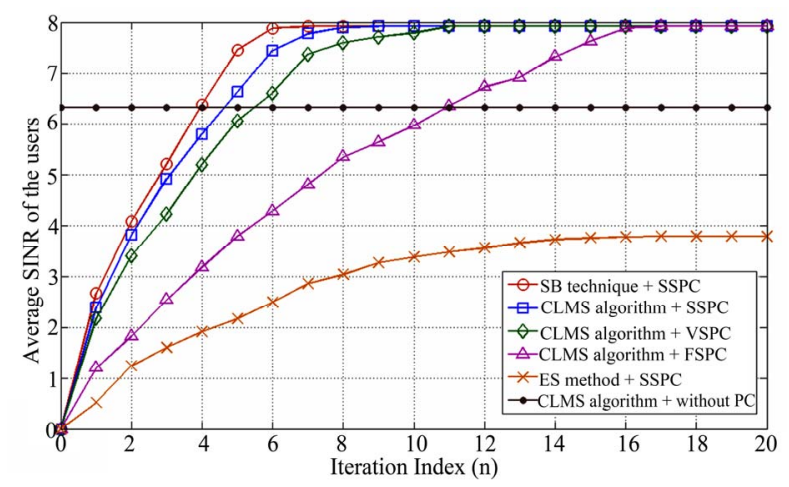

Figure 7. Average SINR of all users versus power control iteration index $(n)$, with maximum power constraint of $1 \mathrm{~W}$. 
gorithm is faster than the VSPC and FSPC algorithms. For example, the SSPC algorithm (for CLMS algorithm) converges in about eight iterations, while VSPC and FSPC algorithms converge in about 11 and 16 iterations, respectively. Also this figure shows that the SSPC algorithm with SB technique converges faster than CLMS and ES methods. Also we observe that the SINR level achieved is below the target SINR value for ES method, because in this case the interference is higher than the other methods.

Figure 8 shows the comparison of TTP usage versus the power control iteration index $(n)$, as Figure 7. But in this simulation, we assume that users no have maximum power constraints. Similar to Figure 7, we observe that the ES method never can achieve the target SINR value for all users. Also this figure shows that the SSPC algorithm offers more savings in the TTP as compared the FSPC and VSPC algorithms. Also the TTP for the joint SSPC algorithm and SB technique is lower than other cases. Although not shown in the figure, in case of without power control and because of near-far problem, the target SINR level is not achieved for all users.

Figure 9 presents the average BER versus the signal to noise ratio (SNR) for CLMS algorithm and SB technique and different values of $\sigma_{v}^{2}$. In this figure, we observe that the average BER for the SB technique is lower than the CLMS algorithm. For example, at a SNR of $10 \mathrm{~dB}$ and $\sigma_{v}^{2}=4 \mathrm{~dB}$, the average BER is 0.0011 for CLMS algorithm, while for SB technique is 0.00018 . Also it can be seen that the average BER for $\sigma_{v}^{2}=0 \mathrm{~dB}$ (perfect power control) is lower than the other cases. For example, at a SNR of $10 \mathrm{~dB}$ and for CLMS algorithm, the average BER is 0.0007 for PPC, while for $\sigma_{v}^{2}=8 \mathrm{~dB}$ the average BER is 0.0016 .

Figure 10 presents the average BER versus the number of active users for SNR $=10 \mathrm{~dB}$ and different values of $\sigma_{v}^{2}$ as Figure 9. Similar to Figure 9, we observe that the average BER for $\sigma_{v}^{2}=0 \mathrm{~dB}$ is lower than $\sigma_{v}^{2}=2,4,8 \mathrm{~dB}$. For example, at a BER of 0.001 , the re-

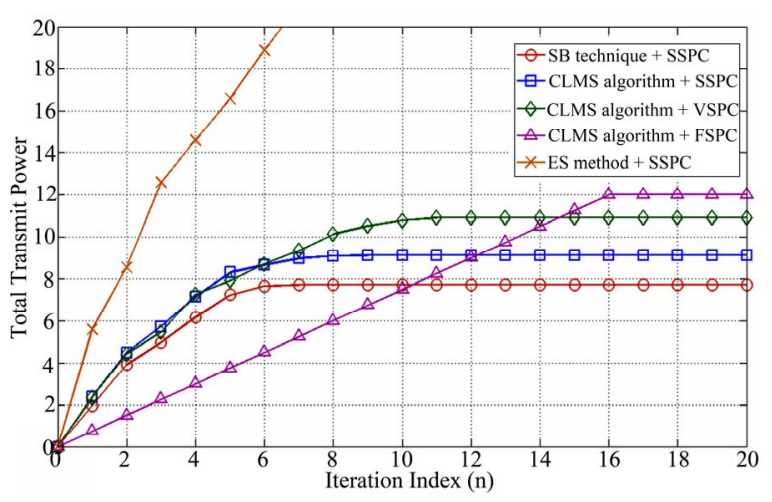

Figure 8. Total transmit power of all users versus power control iteration index $(n)$. No power constraints.

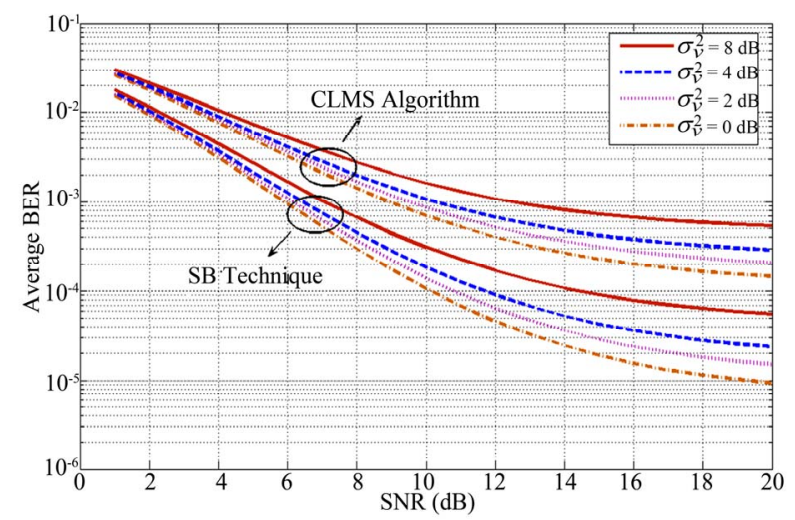

Figure 9. Average BER versus the SNR for the SB technique and CLMS algorithm $(M=15)$.



Figure 10. Average BER versus the number of active users for the SB technique and CLMS algorithm $(S N R=10 \mathrm{~dB})$.

ceiver with CLMS algorithm support $M=18$ users for $\sigma_{v}^{2}=0 \mathrm{~dB}$, while for $\sigma_{v}^{2}=2,4,8 \mathrm{~dB}$ support $M=16,15$, and 12 users, respectively. Accordingly in this case, with $\sigma_{v}^{2}$ from 2 to $8 \mathrm{~dB}$, the system capacity degrades from $6 \%$ to $34 \%$ compared to the case of PPC. We also observe that with $\mathrm{SB}$ technique can achieve lower BER than the CLMS algorithm. For example, at a BER of 0.0001 and $\sigma_{v}^{2}=8 \mathrm{~dB}$, the number of users allowed in the system is $M=15$ users for SB technique, while for CLMS algorithm is $M=5$ users.

Other results displayed in Figure 11 and Figure 12 show the influence of path-loss parameter in USPS $(L)$ and number of antenna elements $(N)$ on the average BER for the CLMS algorithm, SNR $=10 \mathrm{~dB}$, and $\sigma_{v}^{2}$ $=4 \mathrm{~dB}$. In Figure 11, we can observe that, as expected, a decrease in the path-loss parameter entails an increase in the interference and desired signal levels and, therefore using antenna arrays in BS, an improvement in system performance. For example, at a BER of 0.005, capacity is, respectively, 16,29 , and 34 users for $L=0.5$, 0.05 , and $0.01 \mathrm{~d} \mathrm{~B} / \mathrm{m}$. In Figure 12, it is seen that for $L=$ $0.05 \mathrm{~dB} / \mathrm{m}$ and a required average BER of 0.0025 , if $N$ increases from 5 to 15, the number of active users increases by approximately $60 \%$. 




Figure 11. Influence of path-loss parameter $(L)$ on average BER $($ SNR $=10 \mathrm{~dB}, N=10)$.

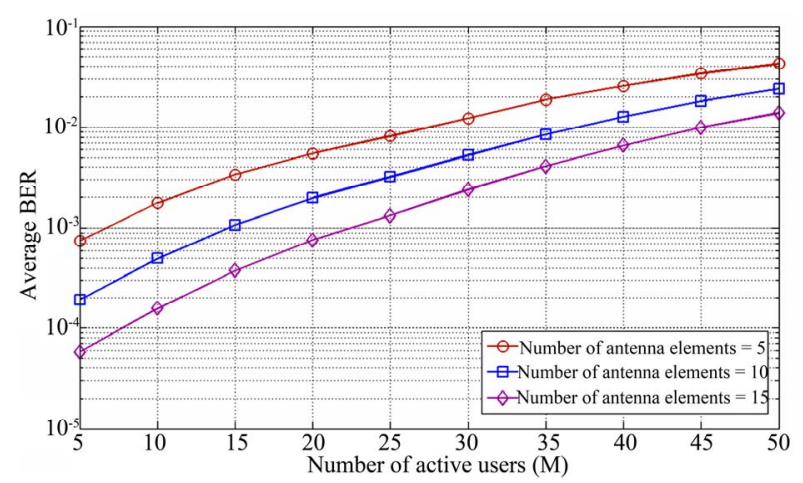

Figure 12. Influence of number of antenna elements $(N)$ on average BER $(\mathrm{SNR}=10 \mathrm{~dB}, L=0.05 \mathrm{~dB} / \mathrm{m})$.

\section{Conclusions}

In this paper, we studied performance of single-cell wireless communication systems in a 2D urban environment with closed-loop power control. Also, we determined the optimum weights of the elements of array antenna with the CLMS algorithm.

Accordingly, we proposed the SSPC algorithm in order to compensate the effects of the near-far problem. It has been shown that, by using antenna arrays at BS, this algorithm will decrease the interference in cell. In addition, the TTP expected by all users is less as compared to the VSPC and FSPC algorithms. Thus, it decreases the BER by allowing the SINR targets for the users to be higher, or by increasing the number of users supportable at a fixed SINR target level. It has also been observed that the TTP in SB technique is less than CLMS algorithm. Also, it has been shown that the convergence speed of the proposed algorithm is increased in comparison with the VSPC and FSPC algorithms. Also, our simulations show that the variations in power level due to PCE have a detrimental effect on system performance.

\section{Acknowledgements}

This research is supported under research project by the
Islamic Azad University, Qazvin Branch, Qazvin, Iran.

\section{References}

[1] J. T. Wang, "Admission Control with Distributed Joint Diversity and Power Control for Wireless Networks," IEEE Transactions on Vehicular Technology, Vol. 58, No. 1, 2009, pp. 409-419.

[2] A. Abrardo and D. Sennati, "On the Analytical Evaluation of Closed-Loop Power-Control Error Statistics in DS-CDMA Cellular Systems," IEEE Transactions on Vehicular Technology, Vol. 49, No. 6, 2000, pp. 2071 -2080 .

[3] L. Carrasco and G. Femenias, "Reverse Link Performance of a DS-CDMA System with both Fast and Slow Power Controlled Users," IEEE Transactions on Wireless Communications, Vol. 7, No. 4, 2008, pp. 1255-1263.

[4] L. Qian and Z. Gajic, "Variance Minimization Stochastic Power Control in CDMA System,” IEEE Transactions on Wireless Communications, Vol. 5, No. 1, 2006, pp. 193202.

[5] M. Rintamaki, H. Koivo and I. Hartimo, "Adaptive Closed-Loop Power Control Algorithms for CDMA Cellular Communication Systems," IEEE Transactions on Vehicular Technology, Vol. 53, No. 6, 2004, pp. 1756 $-1768$.

[6] J. Wang and A. Yu, "Open-Loop Power Control Error in Cellular CDMA Overlay Systems,” IEEE Journal on Selected Areas in Communications, Vol. 19, No. 7, 2001, pp. 1246-1254.

[7] M. Dosaranian-Moghadam, H. Bakhshi, G. Dadashzadeh and P. Rahmati, “Adaptive Beamforming Method Based on Constrained LMS Algorithm for Tracking Mobile User,” IEEE Global Mobile Congress, Shanghai, China, October 2009, pp. 1-6.

[8] M. Dosaranian-Moghadam, H. Bakhshi and G. Dadashzadeh, “Adaptive Beamforming Method Based on Closed -Loop Power Control for DS-CDMA Receiver in Multipath Fading Channel," Accepted for publication in the IEEE International Conference on Communication Systems, Singapore, 17-20 November 2010.

[9] M. Dosaranian-Moghadam, H. Bakhshi and G. Dadashzadeh, "Interference Management for DS-CDMA Systems through Closed-Loop Power Control, Base Station Assignment, and Beamforming,” Journal of Wireless Sensor Network, Vol. 2, No. 6, 2010, pp. 472-482.

[10] M. Dosaranian-Moghadam, H. Bakhshi, G. Dadashzadeh and M. Godarzvand-Chegini, "Joint Base Station Assignment, Power Control Error, and Adaptive Beamforming for DS-CDMA Cellular Systems in Multipath Fading Channels," Accepted for publication in IEEE Global Mobile Congress, Shanghai, China, 18-19 October 2010.

[11] M. Dosaranian-Moghadam, H. Bakhshi and G. Dadashzadeh, "Joint Centralized Power Control and Cell Sectoring for Interference Management in CDMA Cellular Systems in a 2D Urban Environment," Journal of Wireless Sensor Network, Vol. 2, No. 8, 2010, pp. 599-605. 
[12] F. Rashid-Farrokhi, L. Tassiulas and K. J. Ray-Liu, “Joint Optimal Power Control and Beamforming in Wireless Networks using Antenna Arrays," IEEE Transactions on Communications, Vol. 46, No. 10, 1998, pp. 1313-1324.

[13] M. Z. Shakir and T. S. Durrani, "Narrowband Beamforming Algorithm for Smart Antennas,” International Bhurban Conference on Applied Sciences \& Technology, Islamabad, January 2007, pp. 49-54.

[14] X. Y. Sun, X. H. Lian and J. J. Zhou, "Robust Adaptive Beamforming Based on Maximum Likelihood Estimation," International Conference on Microwave and Millimeter Wave Technology, Nanjing, China, Vol. 3, 2008, pp. 1137-1140.

[15] J. Litva and T. Kwok-Yeung, "Digital Beamforming in Wireless Communications,” Artech House, London, 1996.

[16] S. Haykin, “Adaptive Filter Theory,” 3th Edition, Prentice Hall, New Jersey, 1996.

[17] R. L. Peterson, R. E. Ziemer and D. E. Borth, "Spread-Spectrum Communications," Prentice-Hall, New Jersey, 1995.

[18] A. Yener, R. D. Yates and S. Ulukus, "Interference Management for CDMA Systems through Power Control, Multiuser Detection, and Beamforming," IEEE Transactions on Communications, Vol. 49, No. 9, 2001, pp. 1227 -1239 .

[19] S. Kandukuri and S. Boyd, "Optimal Power Control in Interference-Limited Fading Wireless Channels with Outage Probability Specifications," IEEE Transactions on Wireless Communications, Vol. 1, No. 1, 2002, pp. 46-55.
[20] S. Grandhi, R. Vijayan and D. Goodman, "Centralized Power Control in Cellular Radio Systems,” IEEE Transactions on Vehicular Technology, Vol. 42, No. 4, 1993, pp. 466-468.

[21] J. Zander, "Distributed Cochannel Interference Control in Cellular Radio Systems,” IEEE Transactions on Vehicular Technology, Vol. 44, No. 3, 1992, pp. 305-311.

[22] S. Grandhi, R. Vijayan and D.Goodman, "Distributed Power Control in Cellular Radio Systems," IEEE Transactions on Communications, Vol. 42, No. 2/3/4, 1994, pp. 226-228.

[23] A. Kurniawan, "Effect of Feedback Delay on Fixed Step and Variable Step Power Control Algorithm in CDMA Systems," IEEE International Conference on Communication Systems, Singapore, Vol. 2, 2002, pp. 1096-1100.

[24] J. M. Romero-Jerez, C. Tellez-Labao and A. Diaz-Estrella, "Effect of Power Control Imperfections on the Reverse Link of Cellular CDMA Networks under Multipath Fading," IEEE Transactions on Vehicular Technology, Vol. 53, No.1, 2004, pp. 61-71.

[25] B. Allen and M. Beach, "On the Analysis of Switched-Beam Antennas for the W-CDMA Downlink,” IEEE Transactions on Vehicular Technology, Vol. 53, No. 3, 2004, pp. 569-578.

[26] G. E. Corazza, G. De Maio and F. Vatalaro, "CDMA Cellular Systems Performance with Fading, Shadowing, and Imperfect Power Control," IEEE Transactions on Vehicular Technology, Vol. 47, No. 2, 1998, pp. 450-459. 